\title{
Osculatory Interpolation in the Complex Plane
}

\author{
Herbert E. Salzer ${ }^{1}$ \\ Tables of coefficients to facilitate osculatory $n$-point interpolation $(n=2(1) 7)$ in the complex \\ plane are given.
}

The writer has described in a previous article [1] ${ }^{2}$ a new method for osculatory interpolation that can be applied also in the complex plane when one has an analytic function $f(z), z=x+i y$, which is known for arguments $z_{k}$ at equal intervals along any straight line in the $z$-plane. However, for the tabular arguments $z_{k}=x_{k}+i y_{k}$ in the form of a Cartesian grid of length $h$, greater accuracy may be had by basing the Hermite osculatory interpolation formula upon values of $f\left(z_{k}\right)$ and $f^{\prime}\left(z_{k}\right)$ at points $z_{k}$, which are closer together and not necessarily lying upon a straight line (except, of course, in the 2-point case). For a detailed discussion of the Hermite osculatory interpolation formula, the reader is referred to the previous article [1]. This present paper merely aims to supplement that article which was intended primarily for real functions, by giving here the corresponding auxiliary quantities, $a_{k}$ and $b_{k}$, which are suited better for complex interpolation. Whereas the quantities, $a_{k}$ and $b_{k}$, for real interpolation were tabulated up to the 11-point case (21st-degree accuracy), this present tabulation for complex interpolation does not go beyond the 7-point case (13th-degree accuracy), which is more than adequate for most of the practical problems that would arise.

To interpolate for $f(z)$, where $z=z_{0}+P h$ and $P \equiv p+i q$ is now complex, we choose the $z_{k}=z_{0}+k h$ for $k$ equal to certain small complex integers and such that $z_{0}$ will always be the lower left corner point of the configuration of points, $z_{0}$ and $z_{k}$. Also, in almost every example, $|p| \leq 1,|q| \leq 1$, so that the point $z$ is usually not outside the square whose corners are $z_{0}, z_{1}, z_{i}$, and $z_{1+i}$. For each of the $n$-point formulas, $n=2(1) 7$, the points $z_{k}$ are chosen to lie in the following configurations:

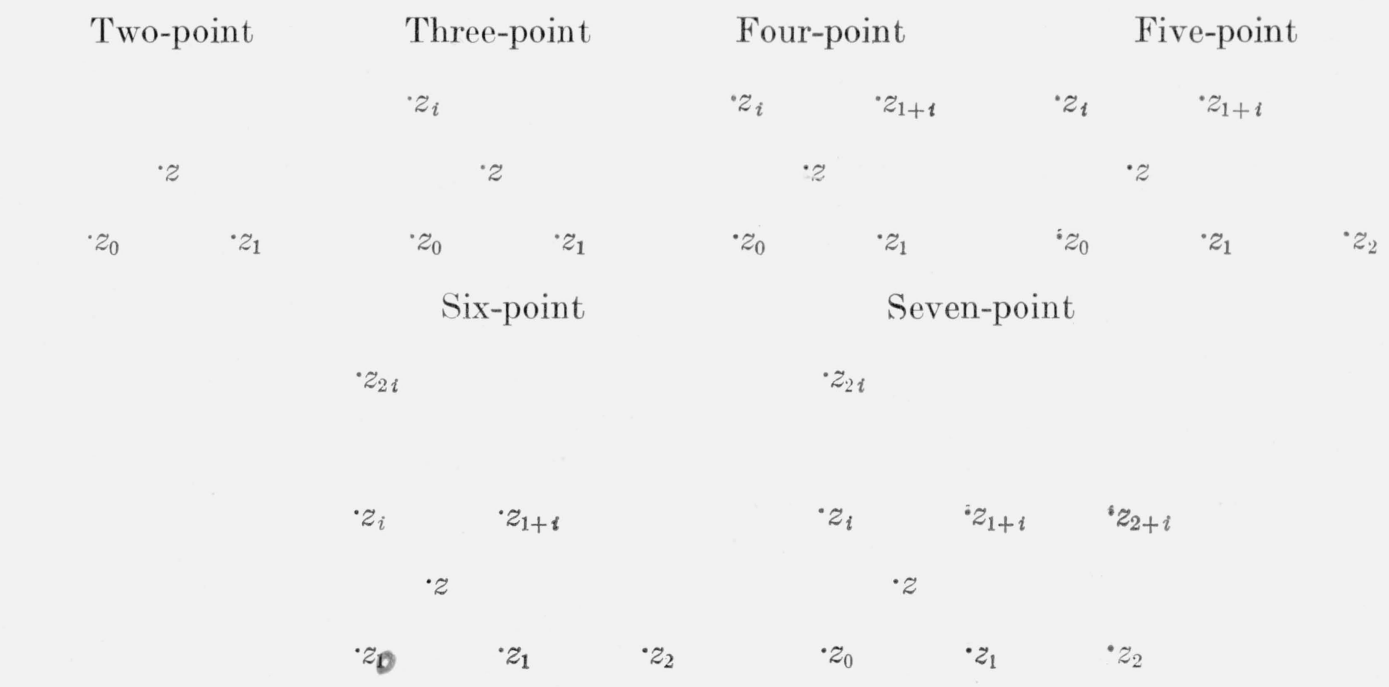

1 Present address: Diamond Ordnance Fuze Laboratory, Department of Defense.

${ }^{2}$ Figures in brackets indicate the literature references at the end of this paper. 
Hermite's $n$-point osculatory interpolation formula for $f(z) \equiv f$, in terms of $f\left(z_{k}\right) \equiv f_{k}$ and $f^{\prime}\left(z_{k}\right) \equiv f^{\prime}{ }_{k}$, is expressible in the concise form

where

$$
f \sim \sum_{k}\left(\alpha_{k} f_{k}+h \beta_{k} f_{k}^{\prime}\right) / \sum_{k} \alpha_{k}
$$

and

$$
\alpha_{k} \equiv a_{k} /(P-k)^{2}+b_{k} /(P-k)
$$

$$
\beta_{k} \equiv a_{k} /(P-k),
$$

the summation being taken for $k$ corresponding to the $n$-points $z_{k}$ of the configuration. The dependence of $\alpha_{k}, \beta_{k}, a_{k}$, and $b_{k}$ upon $n$ is understood. The auxiliary quantities, $a_{k}$ and $b_{k}$, are tabulated below for $n=2(1) 7$ for each of the above $n$-point configurations of points $z_{k}$. The actual definitions of $a_{k}$ and $b_{k}$ are as follows:

and

$$
a_{k}=C(n) /\left\{\Pi_{j}^{\prime}(k-j)\right\}^{2}
$$

$$
b_{k}=-2 C(n)\left\{\frac{d}{d P} \Pi_{j}^{\prime}(P-j)\right\}_{P=k} /\left\{\Pi_{j}^{\prime}(k-j)\right\}^{3}
$$

where the product is taken for $j$ corresponding to the $n-1$ points $z_{j} \neq z_{k}$ of the configuration, and where for each $n, C(n)$ is chosen as the least integer which allows the quantities, $a_{k}$ and $b_{k}$, to appear as complex integers.

Table of $a_{i}$ and $b_{i}$

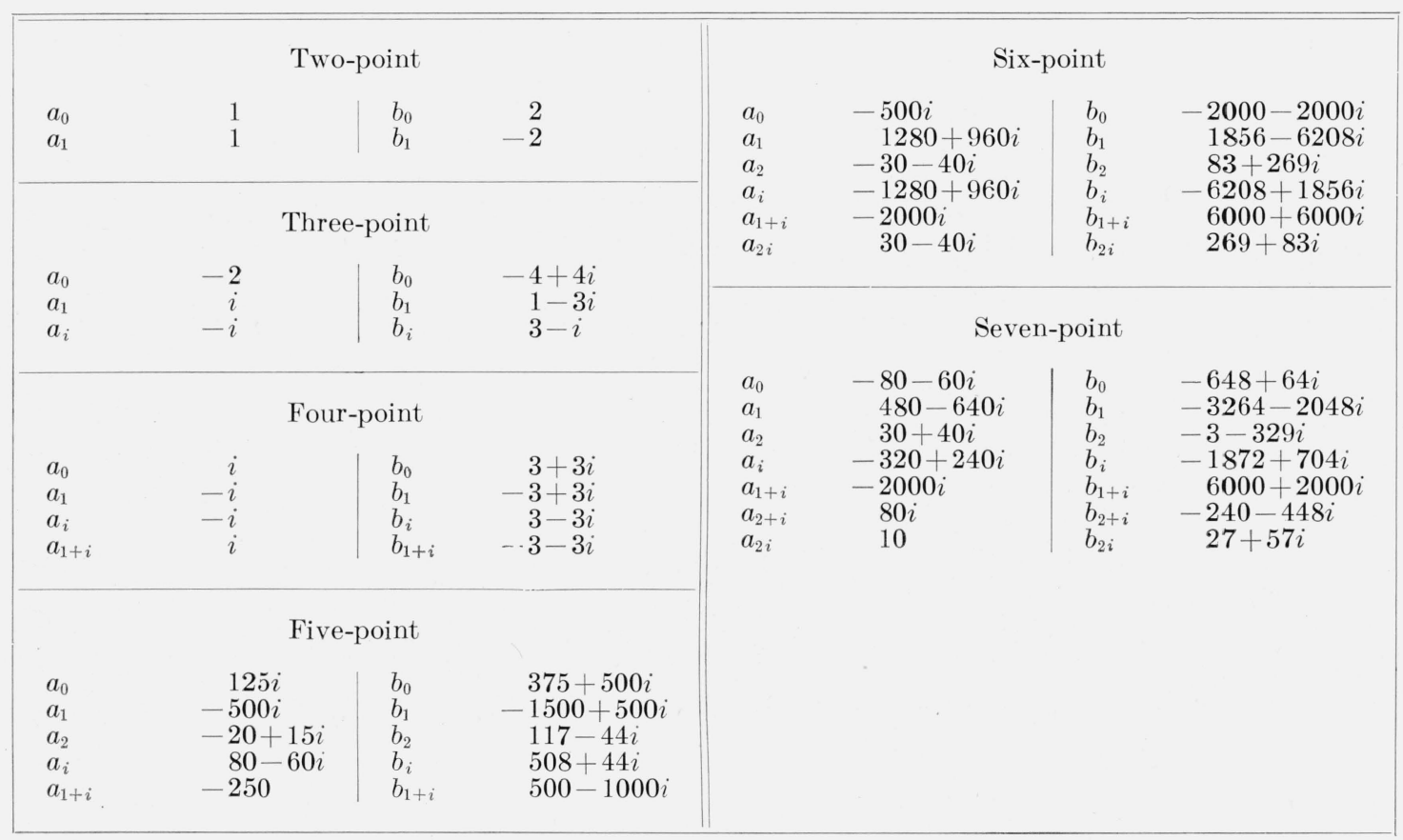

Formulas (1), (2), and (3) for complex osculatory interpolation, which utilize the Cartesian grid, will be especially useful in connection with such tables for complex arguments as (1) logarithm of the gamma function, $\log \Gamma(z)$, together with its derivative the psi function, $\Psi(z)$, 
(2) Bessel functions of the first or second kind giving $J_{0}(z)$ and $J_{1}(z)=-J_{0}^{\prime}(z), Y_{0}(z)$ and $Y_{1}(z)=-Y_{0}^{\prime}(z)$, or linear combinations of them, (3) probability integra $\int_{0}^{z} e^{-u^{2}} d u$ with its integrand, (4) miscellaneous tables of integrals of the more elementary functions where the first derivative or the integrand, although not tabulated, is easy to calculate, namely, the function $\int_{z}^{\infty}\left(e^{-u} / u\right) d u$ and $(5)$ tables of solutions of important linear differential equations, together with their first derivatives [2]. In all such tables, and in many others, the user will find these complex osculatory interpolation formulas to be particularly convenient. They are especially suitable in those cases where the grid length $h$ is too large for sufficiently accurate complex interpolation, using either tables of complex interpolation coefficients $[3,4]$ or formulas corresponding to (1), (2), and (3) above [5], where the tables or formulas involve the use of only the functional values $f_{i}$.

Illustration.-To demonstrate the use of these tables in formulas (1) to (3), consider an example from [2], where the modified Hankel functions $h_{j}(z) \equiv\left(\frac{2}{3} z^{\frac{3}{2}}\right)^{\frac{1}{3}} H_{\frac{1}{3}}^{(j)}\left(\frac{2}{3} z^{\frac{3}{2}}\right), j=1,2$, and their first derivatives are tabulated over a Cartesian grid of length $h=0.1$ in the complex plane. Suppose that it is required to find $h_{1}(1.24579316+0.96155803 i)$, using the four-point osculatory interpolation formula and the tabulated values of $h_{1}, h_{1}^{\prime}$ at $z_{0}=1.2+0.9 i, z_{1}=1.3+0.9 i$, $z_{i}=1.2+1.0 i, z_{1+i}=1.3+1.0 i\left[2\right.$, pp. 21, 23]. Employing (2), (3) and $a_{i}, b_{i}$ from these tables, since $P=0.4579316+0.6155803 i$, one finds that

$$
\begin{aligned}
& \alpha_{0}=i /(0.4579316+0.6155803 i)^{2}+(3+3 i) /(0.4579316+0.6155803 i), \\
& \alpha_{1}=-i /(-0.5420684+0.6155803 i)^{2}+(-3+3 i) /(-0.5420684+0.6155803 i) \text {, } \\
& \alpha_{i}=-i /(0.4579316-0.3844197 i)^{2}+(3-3 i) /(0.45793 \quad 16-0.3844197 i), \\
& \alpha_{1+i}=i /(-0.5420684-0.3844197 i)^{2}+(-3-3 i) /\left(\begin{array}{ll}
-0.54206 & 84-0.3844197 i),
\end{array}\right. \\
& \beta_{0}=i /(0.4579316+0.6155803 i), \beta_{1}=-i /(-0.5420684+0.6155803 i) \text {, } \\
& \beta_{i}=-i /(0.45793 \quad 16-0.3844197 i), \beta_{1+i}=i /\left(\begin{array}{ll}
-0.54206 & 84-0.3844197 i),
\end{array}\right.
\end{aligned}
$$

from which one obtains.

$$
\begin{aligned}
& \alpha_{0}=7.098245538-1.291879023 i, \quad \beta_{0}=1.045766212+0.777947889 i, \\
& \alpha_{1}=6.636540823+0.515813695 i, \quad \beta_{1}=-0.914983814+0.805717487 i \text {, } \\
& \alpha_{i}=9.824153465-1.101478051 i, \quad \beta_{i}=1.075360453-1.280999733 i, \\
& \alpha_{1+i}=8.43081 \quad 1380+1.819869385 i, \quad \beta_{1+i}=-0.87048 \quad 2793-1.227463667 i,
\end{aligned}
$$

Then for (1) we employ the following tabulated values of $h_{1}$ and $h_{1}^{\prime}$ :

$$
\begin{aligned}
& f_{0}=0.19018585-0.19313840 i, \quad f_{0}^{\prime}=0.14952614+0.33229461 i, \\
& f_{1}=0.20311754-0.15966418 i, \quad f_{1}^{\prime}=0.10901235+0.33659231 i \text {, } \\
& f_{i}=0.15891089-0.17847850 i, \quad f_{i}^{\prime}=0.14376 \quad 041+0.29375 \quad 232 i, \\
& f_{1+i}=0.17143575-0.14888108 i, \quad f_{1+i}^{\prime}=0.10669347+0.29764346 i \text {, }
\end{aligned}
$$

and also

so that we have

$$
\sum_{k} \alpha_{k}=31.98975121-0.05767399 i
$$

$h_{1}(1.24579316+0.96155803 i) \equiv f \sim\left[\alpha_{0} f_{0}+\alpha_{1} f_{1}+\alpha_{i} f_{i}\right.$

$$
\left.+\alpha_{1+i} f_{1+i}+0.1\left\{\beta_{0} f_{0}^{\prime}+\beta_{1} f_{1}^{\prime}+\beta_{i} f_{i}^{\prime}+\beta_{1+i} f_{1+i}^{\prime}\right\}\right] / \sum_{k} \alpha_{k},
$$


whose terms are

$$
\begin{aligned}
& {[(7.098245538-1.291879023 i)(0.19018 \quad 585-0.19313 \quad 840 i)} \\
& +\left(\begin{array}{ll}
6.63654 & 0823+0.515813695 i)(0.20311 \quad 754-0.15966 \quad 418 i)
\end{array}\right. \\
& +\left(\begin{array}{lllll}
9.82415 & 3465-1.10147 & 8051 i)(0.15891 & 089-0.17847 & 850 i
\end{array}\right) \\
& +(8.430811380+1.819869385 i)(0.17143 \quad 575-0.14888108 i) \\
& +0.1\{(1.04576 \quad 6212+0.77794 \quad 7889 i)(0.14952614+0.33229461 i) \\
& +(-0.914983814+0.80571 \quad 7487 i)(0.10901235+0.33659231 i)
\end{aligned}
$$

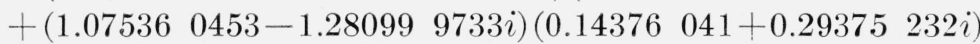

$$
\begin{aligned}
& +(-0.870482793-1.22746 \quad 3667 i)(0.10669347+0.29764 \quad 346 i)\}] \\
& \div(31.98975121-0.05767399 i) \text {, }
\end{aligned}
$$

or after multiplying,

$$
\begin{aligned}
& {[(1.100474414-1.616640896 i)+(1.430354817-0.954847040 i)} \\
& +(1.364574820-1.928437032 i)+(1.716286592-0.943197631 i) \\
& +0.1\{(-0.102138505+0.463826021 i)+(-0.370942846-0.220143359 i) \\
& +(0.530890903+0.131732581 i)+(0.27247 \quad 1703-0.390055868 i)\}] \\
& \div\left(\begin{array}{lll}
31.98975 & 121-0.05767 & 399 i
\end{array}\right) \\
& =\left(\begin{array}{llll}
5.64471 & 8767-5.44458 & 6662 i
\end{array}\right) /\left(\begin{array}{lll}
31.98975 & 121-0.05767 & 399 i
\end{array}\right) \text {, }
\end{aligned}
$$

or finally,

$$
0.17676025-0.16987916 i
$$

which is correct to within a unit in the last place, as was seen from the independent calculation of $h_{1}(1.24579316+0.96155803 i)$ from the Taylor series around $z_{0}=1.2+0.9 i$.

[1] H. E. Salzer, New formulas for facilitating osculatory interpolation, J. Research NBS 52, 211 (1954) RP2491.

[2] Harvard Computation Laboratory, Tables of the modified Hankel functions of order one-third and of their derivatives (Harvard University Press, Cambridge, Mass., 1945).

[3] A. N. Lowan and H. E. Salzer, Coefficients for interpolation within a square grid in the complex plane. J. Math. Phys. 23, 156 (1944).

[4] H. E. Salzer, Coefficients for complex quartic, quintic, and sextic interpolation within a square grid, J. Math. Phys. 27, 136 (1948).

[5] H. E. Salzer, Formulas for complex Cartesian interpolation of higher degree, J. Math. Phys. 28, 200 (1949).

Washington, August 6, 1954. 